PROCEEDINGS OF THE

AMERICAN MATHEMATICAL SOCIETY

Volume 126, Number 4, April 1998, Pages 1127-1132

S 0002-9939(98)04347-0

\title{
NEW FIXED POINT THEOREMS FOR A FAMILY OF MAPPINGS AND APPLICATIONS TO PROBLEMS ON SETS WITH CONVEX SECTIONS
}

\author{
KUNQUAN LAN AND JEFFREY WEBB
}

(Communicated by Palle E. T. Jorgensen)

\begin{abstract}
Some new fixed point theorems for a family of mappings are obtained and applied to problems on sets with convex sections that were first studied by Ky Fan.
\end{abstract}

\section{INTRODUCTION}

In this paper we shall employ a partition of unity and Tychonoff's fixed point theorem to prove some new fixed point theorems for a family of mappings defined in product spaces. Our theorems are particularly well suited to treat problems on sets with convex sections. It is well known that the latter have applications in minimax inequalities of von Neumann type and in game theory, for example, the existence of Nash points.

Let $I$ be an index set and $\left\{X_{i}\right\}_{i \in I}$ be a family of convex sets each in a Hausdorff topological vector space. Write $X=\prod_{i \in I} X_{i}$ and let $\left\{A_{i}\right\}_{i \in I}$ be a family of subsets of $X$. Then the problem on sets with convex sections is to find suitable conditions on $\left\{A_{i}\right\}_{i \in I}$ such that the family has a nonempty intersection.

When $I$ is a finite set and $X$ is compact, Ky Fan [Fan] first imposed a convexity and an openness condition on each $A_{i}$ which assured that the family $\left\{A_{i}\right\}_{i \in I}$ has a nonempty intersection. A new proof of Fan's result was given by Browder [Br] using his fixed point theorem. The idea of Browder was employed in [T] and [T.T] to improve Fan's result, but this fixed point technique cannot be applied to treat the case when $I$ is infinite. The difficulty is that the intersection of infinitely many open sets may not be open.

Fan's result was generalized in two directions. Ma [Ma] employed a partition of unity and an intersection result in [Fan3] (one could also use Tychonoff's fixed point theorem) to generalize Fan's result to the case when $I$ is infinite. Then Fan [Fan2] generalized Ma's result by considering two families of subsets to relax the convexity and openness conditions. Fan's latter result was generalized by Shih and Tan [S.T] to noncompact settings.

On the other hand, Fan [Fan2] generalized his original result by relaxing compactness and convexity conditions and showed that the intersection of some subfamily

Received by the editors September 23, 1996.

1991 Mathematics Subject Classification. Primary 46A55, 47H04, 47H10, 54H25; Secondary 49J40.

Key words and phrases. Fixed point, partition of unity, sets with convex sections. 
of $\left\{A_{i}\right\}_{i \in I}$ was not empty (see Theorem 15 in [Fan2]). The main idea is to change the question into an intersection problem for $K K M$ maps. Fan's idea was then employed by Shih and Tan [S.T] to improve Fan's result to two families of subsets with a different compactness condition. However, this method cannot be applied to treat the case when $I$ is infinite due to the fact that the union of infinitely many closed sets may not be closed. Hence, when $I$ is an infinite set, the problem has not been solved until now.

In this paper we shall first consider the problem of existence of fixed points for a family of mappings $\left\{\psi_{i}\right\}_{i \in I}$, where $\psi_{i}: X^{i} \rightarrow 2^{X_{i}}$, that is, to find $x \in X$ such that $x_{i} \in \psi_{i}\left(x^{i}\right)$ for all $i \in I$. (The precise meanings of these symbols will be given later). Since the space $X$ involved in our fixed point theorems may not be compact, we employ a special technique in the proof of our main fixed point theorem to utilize a partition of unity and Tychonoff's fixed point theorem.

As applications of our new fixed point theorems, we deduce new results on sets with convex sections. We show that all the above generalizations of Fan's result on sets with convex sections can be unified in a more general result which applies whether the index set $I$ is finite or infinite. Furthermore, all of the previous compactness conditions used in [Fan2] and [S.T] are replaced by a weaker compactness condition. Hence, our results on sets with convex sections not only unify the previous results but also are very general.

\section{FiXed point theorems And Results on Sets With CONVEX SECtions}

Throughout this section, let $I$ be an index set and for each $i \in I$ let $E_{i}$ be a Hausdorff topological vector space. Let $X_{i}$ be a nonempty convex subset of $E_{i}$, $X=\prod_{i \in I} X_{i}$ and $X^{i}=\prod_{j \neq i, j \in I} X_{j}$. We write $X=X_{i} \otimes X^{i}$. Then, for each fixed $i \in I$ and each $x \in X$, we write $x=\left(x_{i}, x^{i}\right)$, where $x_{i} \in X_{i}$ and $x^{i} \in X^{i}$.

Assume that $E$ is a Hausdorff topological vector space and $Y$ is a nonempty convex subset in $E$. We denote by $2^{Y}$ the family of all subsets of $Y$ and by co $B$ the convex hull of a set $B$. Let $G: Y \rightarrow 2^{Y}$ be a multivalued map. We define $G^{-1}$, $G^{*}: Y \rightarrow 2^{Y}$ by $G^{-1}(y)=\{x \in Y: y \in G(x)\}$ and $G^{*}(y)=\{x \in Y: y \notin G(x)\}$, respectively. Some properties of the above maps can be found in Lemma 3.2 of [Lan]. We shall directly apply these properties.

We start with the following new fixed point theorem for a family of mappings defined in product spaces, which is a key result in this paper.

Theorem 2.1. For each $i \in I$, let $\psi_{i}: X^{i} \rightarrow 2^{X_{i}}$ be a map. Assume that the following conditions hold.

$\left(h_{1}\right)$ For each $x^{i} \in X^{i}, \psi_{i}\left(x^{i}\right) \neq \emptyset$.

$\left(h_{2}\right)$ For each $x^{i} \in X^{i}, \psi_{i}\left(x^{i}\right)$ is convex.

$\left(h_{3}\right)$ For each $i \in I$ and each $y_{i} \in X_{i}, \psi_{i}^{-1}\left(y_{i}\right)$ is open in $X^{i}$.

$\left(h_{4}\right)$ If $X^{i}$ is not compact, assume that there exist a nonempty compact convex subset $X_{i}^{0}$ of $X_{i}$ and a nonempty compact subset $D(i)$ of $X^{i}$ such that $X_{i}^{0} \cap \psi_{i}\left(x^{i}\right) \neq$ $\emptyset$ for each $x^{i} \in X^{i} \backslash D(i)$.

Then there exists $x \in X$ such that $x_{i} \in \psi_{i}\left(x^{i}\right)$ for all $i \in I$.

Proof. For each $i \in I$, we define a map $\phi_{i}: X_{i} \rightarrow 2^{X^{i}}$ by $\phi_{i}\left(x_{i}\right)=\psi_{i}^{*}\left(x_{i}\right)$. Then $\phi_{i}$ satisfies the following conditions.

(a) For each $x_{i} \in X_{i}, \phi_{i}\left(x_{i}\right)$ is closed in $X^{i}$ by $\left(h_{3}\right)$.

(b) For each $i \in I, \bigcap_{x_{i} \in X_{i}^{0}} \phi_{i}\left(x_{i}\right)$ is compact in $X^{i}$. 
In fact, if $X^{i}$ is compact, $\bigcap_{x_{i} \in X_{i}^{0}} \phi_{i}\left(x_{i}\right)$ is compact since $\bigcap_{x_{i} \in X_{i}^{0}} \phi_{i}\left(x_{i}\right)$ is closed in $X^{i}$ by $(a)$. If $X^{i}$ is not compact, $\bigcap_{x_{i} \in X_{i}^{0}} \phi_{i}\left(x_{i}\right) \subset D(i)$ by $\left(h_{4}\right)$ and thus is compact.

(c) For each $i \in I, \bigcap_{x_{i} \in X_{i}} \phi_{i}\left(x_{i}\right)=\emptyset$ by $\left(h_{1}\right)$.

Now, we prove that there exist $a_{i 1}, \ldots, a_{i l_{i}} \in X_{i}$ such that

$$
\bigcap_{x_{i} \in X_{i}^{0}} \phi_{i}\left(x_{i}\right) \cap\left(\bigcap_{k=1}^{l_{i}} \phi_{i}\left(a_{i k}\right)\right)=\emptyset .
$$

In fact, if not, then for every finite set $\left\{y_{1}, \ldots, y_{n}\right\} \subset X_{i}$,

$$
\bigcap_{x_{i} \in X_{i}^{0}} \phi_{i}\left(x_{i}\right) \cap\left(\bigcap_{j=1}^{n} \phi_{i}\left(y_{j}\right)\right) \neq \emptyset .
$$

Let $G(y)=\bigcap_{x_{i} \in X_{i}^{0}} \phi_{i}\left(x_{i}\right) \cap \phi_{i}(y)$ for $y \in X_{i}$. Then the family $\left\{G(y): y \in X_{i}\right\}$ has the finite intersection property. Note that $G(y)$ is compact for each $y \in X_{i}$ by $(a)$ and $(b)$. It follows that $\bigcap_{y \in X_{i}} G(y) \neq \emptyset$, and thus $\bigcap_{y \in X_{i}} \phi_{i}(y) \neq \emptyset$, which contradicts $(c)$.

By (2.1) we have

$$
\bigcup_{x_{i} \in X_{i}^{0}} \psi_{i}^{-1}\left(x_{i}\right) \cup\left(\bigcup_{k=1}^{l_{i}} \psi_{i}^{-1}\left(a_{i k}\right)\right)=X^{i} .
$$

Let $F_{i}=\operatorname{co}\left(X_{i}^{0} \cup\left\{a_{i 1}, \ldots, a_{i l_{i}}\right\}\right)$. Then $F_{i}$ is compact in $X_{i}$. For each $i \in I$, let $F^{i}=\prod_{j \neq i, j \in I} F_{j}$. Then $F^{i}$ is a compact subset of $X^{i}$ for each $i \in I$. By (2.2) we have

$$
F^{i} \subset \bigcup_{x_{i} \in X_{i}^{0}} \psi_{i}^{-1}\left(x_{i}\right) \cup\left(\bigcup_{k=1}^{l_{i}} \psi_{i}^{-1}\left(a_{i k}\right)\right) .
$$

Since $F^{i}$ is compact, there exist $b_{i 1}, \ldots, b_{i t_{i}} \in X_{i}$ such that

$$
F^{i} \subset \bigcup_{j=1}^{t_{i}} \psi_{i}^{-1}\left(b_{i j}\right) \cup\left(\bigcup_{k=1}^{l_{i}} \psi_{i}^{-1}\left(a_{i k}\right)\right) .
$$

Let $\left\{c_{i 1}, \ldots, c_{i n_{i}}\right\}=\left\{a_{i 1}, \ldots, a_{i l_{i}}, b_{i 1}, \ldots, b_{i t_{i}}\right\}$. We rewrite (2.4) as follows.

$$
F^{i} \subset \bigcup_{k=1}^{n_{i}} \psi_{i}^{-1}\left(c_{i k}\right)
$$

Let $K_{i}=\operatorname{co}\left\{c_{i 1}, \ldots, c_{i n_{i}}\right\}, K=\prod_{i \in I} K_{i}$ and $K^{i}=\prod_{j \neq i, j \in I} K_{j}$. We write $K=$ $K_{i} \otimes K^{i}$. Denote by $S_{i}$ the vector subspace of $E_{i}$ generated by $K_{i}$. Then $S_{i}$ is locally convex since it is a finite dimensional subspace. We note that $K$ and $K^{i}$ are compact in $\prod_{i \in I} S_{i}$ and $\prod_{j \in I, j \neq i} S_{j}$, respectively, and $K^{i} \subset F^{i}$ for each $i \in I$. For each $i \in I$, let $O_{i k}=\psi_{i}^{-1}\left(c_{i k}\right) \cap K^{i}$ for each $k \in\left\{1, \ldots, n_{i}\right\}$. Then $O_{i k}$ is open in $K^{i}$ for each $k \in\left\{1, \ldots, n_{i}\right\}$, and $K^{i}=\bigcup_{k=1}^{n_{i}} O_{i k}$.

Let $\left\{f_{i 1}, \ldots, f_{i n_{i}}\right\}$ be a non-negative continuous partition of unity subordinate to this open covering of $K^{i}$. Then

$$
f_{i k}\left(x^{i}\right)=0 \quad \text { for } \quad x^{i} \in K^{i} \backslash O_{i k} \quad \text { and } \quad \sum_{k=1}^{n_{i}} f_{i k}\left(x^{i}\right)=1 \quad \text { for } \quad x^{i} \in K^{i} .
$$


Define a continuous map $g_{i}: K^{i} \rightarrow 2^{K_{i}}$ by $g_{i}\left(x^{i}\right)=\sum_{k=1}^{n_{i}} f_{i k}\left(x^{i}\right) c_{i k}$. Note that $f_{i k}\left(x^{i}\right) \neq 0$ implies $x^{i} \in O_{i k}$. This implies $x^{i} \in \psi_{i}^{-1}\left(c_{i k}\right)$, and thus $c_{i k} \in \psi_{i}\left(x^{i}\right)$. It follows from $\left(h_{2}\right)$ that $g_{i}\left(x^{i}\right) \in \psi_{i}\left(x^{i}\right)$.

Define a map $f: K \rightarrow K$ by $f(x)=\left(g_{i}\left(x^{i}\right)\right)_{i \in I}$. Since for each $x \in K$ we have $x^{i} \in K^{i}$ and $g_{i}\left(x^{i}\right) \in K_{i}$, it follows that $f$ is well-defined and continuous. By Tychonoff's fixed point theorem [Ty], $f$ has a fixed point $x=\left(x_{i}\right)_{i \in I} \in K$. This implies $x_{i} \in g_{i}\left(x^{i}\right)$ for all $i \in I$. Since $g_{i}\left(x^{i}\right) \subset \psi_{i}\left(x^{i}\right)$ for each $i \in I$, we have $x_{i} \in \psi_{i}\left(x^{i}\right)$ for all $i \in I$.

Remark 2.1. The condition $\left(h_{4}\right)$ is equivalent to the following condition.

$\left(C_{1}\right)$ If $X^{i}$ is not compact, assume that there exist a nonempty compact convex subset $X_{i}^{0}$ of $X_{i}$ and a nonempty compact subset $D(i)$ of $X^{i}$ such that for each $x^{i} \in X^{i} \backslash D(i)$ there exists $x_{i}^{0} \in X_{i}^{0}$ such that $x_{i}^{0} \in \psi_{i}\left(x^{i}\right)$.

The following conditions are special cases of $\left(h_{4}\right)$.

$\left(C_{2}\right)$ If $X$ is not compact, assume that there exist a nonempty compact convex subset $K$ of $X$ and a nonempty compact subset $D$ of $X$ such that for each $x \in X \backslash D$ there exists $y \in K$ such that $y_{i} \in \psi_{i}\left(x^{i}\right)$ for all $i \in I$.

To see that $\left(C_{2}\right)$ implies $\left(h_{4}\right)$, we show that $\left(C_{2}\right)$ implies $\left(C_{1}\right)$. We define maps $p_{i}: X \rightarrow X_{i}$ and $p^{i}: X \rightarrow X^{i}$ by $p_{i}(x)=x_{i}$ and $p^{i}(x)=x^{i}$. Let $D(i)=P^{i}(D)$ and $X_{i}^{0}=p_{i}(K)$. Now, for each $i \in I$ and each $x^{i} \in X^{i} \backslash D(i)$ (i.e., $x^{i} \notin D(i)$ ) and for any fixed $x_{i} \in X_{i}$, we have $x=\left(x_{i}, x^{i}\right) \notin D$. By $\left(C_{2}\right)$ there exists $y \in K$ such that $y_{i} \in \psi_{i}\left(x^{i}\right)$, that is, $y_{i} \in X_{i}^{0}$ and $y_{i} \in \psi_{i}\left(x^{i}\right)$. Hence $\left(C_{1}\right)$ holds.

$\left(C_{3}\right)$ If $X^{i}$ is not compact, assume that there exists a nonempty compact subset $D(i)$ of $X^{i}$ such that $\bigcap_{x^{i} \in X^{i} \backslash D(i)} \psi_{i}\left(x^{i}\right) \neq \emptyset$.

$\left(C_{4}\right)$ If $X^{i}$ is not compact, assume that there exist $z_{i} \in X_{i}$ and a nonempty compact subset $D(i)$ of $X^{i}$ such that $\psi_{i}^{*}\left(z_{i}\right) \subset D(i)$.

$\left(C_{5}\right)$ If $X$ is not compact, assume that there exists a nonempty compact subset $D \subset X$ such that $D \cap \prod_{i \in I} \psi_{i}\left(y^{i}\right) \neq \emptyset$ for $y \in X \backslash D$.

In fact, $\left(C_{3}\right)$ and $\left(C_{4}\right)$ are equivalent, and $\left(C_{5}\right)$ implies $\left(C_{4}\right)$.

The following result is a slight generalization of Theorem 2.1.

Theorem 2.2. For each $i \in I$, let $\phi_{i}: X^{i} \rightarrow 2^{X_{i}}$ be a map. Assume that the following conditions hold.

$\left(H_{1}\right)$ For each $x^{i} \in X^{i}, \phi_{i}\left(x^{i}\right) \neq \emptyset$.

$\left(H_{2}\right)$ For each $i \in I$ and each $y_{i} \in X_{i}, \phi_{i}^{-1}\left(y_{i}\right)$ is open in $X^{i}$.

$\left(H_{3}\right)$ If $X^{i}$ is not compact, assume that there exist a nonempty compact convex subset $X_{i}^{0}$ of $X_{i}$ and a nonempty compact subset $D(i)$ of $X^{i}$ such that $X_{i}^{0} \cap$ $\operatorname{co} \phi_{i}\left(x^{i}\right) \neq \emptyset$ for each $x^{i} \in X^{i} \backslash D(i)$.

Then there exists $x \in X$ such that $x_{i} \in \operatorname{co} \phi_{i}\left(x^{i}\right)$ for all $i \in I$.

Proof. For each $i \in I$, we define a map $\psi_{i}: X^{i} \rightarrow 2^{X_{i}}$ by $\psi_{i}\left(x^{i}\right)=\operatorname{co} \phi_{i}\left(x^{i}\right)$. Then it is easy to verify that $\psi_{i}$ satisfies $\left(h_{1}\right),\left(h_{2}\right)$ and $\left(h_{4}\right)$. By Lemma 5.1 in [Y.P] we see that $\left(H_{2}\right)$ implies $\psi_{i}$ satisfies $\left(h_{3}\right)$. The result follows from Theorem 2.1.

By Theorem 2.2 we obtain the following result on sets with convex sections.

Theorem 2.3. Let $\left\{A_{i}\right\}_{i \in I}$ be a family of subsets of $X$ such that the following conditions hold.

$\left(S_{1}\right)$ For each $i \in I$ and each $x^{i} \in X^{i},\left\{x_{i} \in X_{i}:\left(x_{i}, x^{i}\right) \in A_{i}\right\} \neq \emptyset$.

$\left(S_{2}\right)$ For each $i \in I$ and each $x_{i} \in X_{i}$, the section $\left\{x^{i} \in X^{i}:\left(x_{i}, x^{i}\right) \in A_{i}\right\}$ is open in $X^{i}$. 
$\left(S_{3}\right)$ If $X^{i}$ is not compact, assume that there exist a nonempty compact convex subset $X_{i}^{0}$ of $X_{i}$ and a nonempty compact subset $D(i)$ of $X^{i}$ such that for each $x^{i} \in X^{i} \backslash D(i), X_{i}^{0} \cap \operatorname{co}\left\{x_{i} \in X_{i}:\left(x_{i}, x^{i}\right) \in A_{i}\right\} \neq \emptyset$.

Then there exists $x \in X$ such that $x_{i} \in \operatorname{co}\left\{x_{i} \in X_{i}:\left(x_{i}, x^{i}\right) \in A_{i}\right\}$ for all $i \in I$.

Proof. For each $i \in I$, we define a map $\phi_{i}: X^{i} \rightarrow 2^{X_{i}}$ by

$$
\phi_{i}\left(x^{i}\right)=\left\{x_{i} \in X_{i}:\left(x_{i}, x^{i}\right) \in A_{i}\right\} .
$$

Then it is easy to verify that $\left\{\phi_{i}\right\}_{i \in I}$ satisfies all the condition of Theorem 2.2. The result follows.

Remark 2.2. The following conditions $\left(C_{2}^{\prime}\right)$ and $\left(C_{5}^{\prime}\right)$ are special cases of $\left(S_{3}\right)$.

$\left(C_{2}^{\prime}\right)$ If $X$ is not compact, assume that there exist a nonempty compact convex subset $K$ of $X$ and a nonempty compact subset $D$ of $X$ such that for each $x \in X \backslash D$ there exists $y \in K$ such that $\left(y_{i}, x^{i}\right) \in A_{i}$ for all $i \in I$.

We show $\left(C_{2}^{\prime}\right)$ implies $\left(S_{3}\right)$. Assume that $\left(C_{2}^{\prime}\right)$ holds. For each $i \in I$, let $\psi_{i}\left(x^{i}\right)=$ $\left\{x_{i} \in X_{i}:\left(x_{i}, x^{i}\right) \in A_{i}\right\}$, and let $D(i)$ and $X_{i}^{0}$ be as in the proof of $\left(C_{2}\right)$ implies $\left(C_{1}\right)$. Then $\left\{\psi_{i}\right\}_{i \in I}$ satisfies $\left(C_{2}\right)$. Since $\left(C_{2}\right)$ implies $\left(C_{1}\right)$ and $\left(C_{1}\right)$ implies $\left(h_{4}\right)$, we have $X_{i}^{0} \cap \psi_{i}\left(x^{i}\right) \neq \emptyset$ for each $x^{i} \in X^{i} \backslash D(i)$; that is, $X_{i}^{0} \cap\left\{x_{i} \in X_{i}:\left(x_{i}, x^{i}\right) \in A_{i}\right\} \neq \emptyset$ for each $x^{i} \in X^{i} \backslash D(i)$. This implies $\left(S_{3}\right)$.

$\left(C_{5}^{\prime}\right)$ If $X$ is not compact, assume that there exists a nonempty compact subset $D \subset X$ such that $D \cap \prod_{i \in I} \psi_{i}\left(y^{i}\right) \neq \emptyset$ for $y \in X \backslash D$, where $\psi_{i}\left(x^{i}\right)=\left\{x_{i} \in X_{i}\right.$ : $\left.\left(x_{i}, x^{i}\right) \in A_{i}\right\}$.

Using Theorem 2.3, we obtain the following general result on sets with convex sections for two families of subsets.

Theorem 2.4. Assume that all the conditions of Theorem 2.3 hold. Let $\left\{B_{i}\right\}_{i \in I}$ be a family of subsets of $X$ such that the following condition holds.

$(H)$ For each $x \in X$ there exists a subset $I(x) \subset I$ such that for $i \in I(x)$,

$$
\operatorname{co}\left\{y_{i} \in X_{i}:\left(y_{i}, x^{i}\right) \in A_{i}\right\} \subset\left\{y_{i} \in X_{i}:\left(y_{i}, x^{i}\right) \in B_{i}\right\}
$$

Then there exists $x \in X$ such that $\bigcap_{i \in I(x)} B_{i} \neq \emptyset$.

Proof. There exists $x \in X$ such that $x_{i} \in \operatorname{co}\left\{x_{i} \in X_{i}:\left(x_{i}, x^{i}\right) \in A_{i}\right\}$ for all $i \in I$ by Theorem 2.3. Hence, for this $x, x_{i} \in\left\{y_{i} \in X_{i}:\left(y_{i}, x^{i}\right) \in B_{i}\right\}$ for $i \in I(x)$. This implies $x \in B_{i}$ for all $i \in I(x)$.

Remark 2.3. Theorem 2.4 generalizes Theorem 15 in [Fan2] and Theorem 5 in [S.T] in the following ways: $(i)$ the set $I$ may be infinite; $(i i)$ the conditions $\left(S_{3}\right)$ are weaker than the condition $(c)$ in Theorem 15 of [Fan2] (see $\left(C_{2}^{\prime}\right)$ in Remark 2.2) and the conditions $\left(d^{\prime \prime}\right)$ of Theorem 5 in [S.T] (see $\left(C_{5}^{\prime}\right)$ in Remark 2.2); and (iii) the condition $(H)$ is weaker than the condition $(d)$ in Theorem 15 of [Fan2] and the condition $(c)$ in Theorem 5 of $[\mathrm{S} . \mathrm{T}]$. Moreover, we see that our method is different from those used in Theorem 15 of [Fan2] and Theorem 5 in [S.T].

Even when $I(x)=I$ for $x \in X$, Theorem 2.4 generalizes Theorem 2 in [S.T]; and when $A_{i}=B_{i}$, Theorem 2.4 generalizes Theorem 16 in [Fan2] and those noted in [Fan2], namely generalizations of Theorem 2 in [Ma] and Theorem 1 in [Fan1].

We can state an analytic formulation of Theorem 2.4, but we only give the following simple result. 
Theorem 2.5. Let $\left\{f_{i}\right\}_{i \in I}: X \rightarrow \mathbb{R}$ be a family of functions and $\left\{t_{i}\right\}_{i \in I}$ a sequence of real numbers. Assume that the following conditions hold.

$\left(s_{1}\right)$ For each $i \in I$ and each $x^{i} \in X^{i}, f_{i}\left(., x^{i}\right)$ is a quasiconcave function on $X_{i}$.

$\left(s_{2}\right)$ For each $i \in I$ and each $x_{i} \in X_{i}, f_{i}\left(x_{i},.\right)$ is a lower semicontinuous function on $X^{i}$. $t_{i}$.

$\left(s_{3}\right)$ For each $i \in I$ and each $x^{i} \in X^{i}$, there exists $y_{i} \in X_{i}$ such that $f_{i}\left(y_{i}, x^{i}\right)>$

$\left(s_{4}\right)$ If $X$ is not compact, assume that there exist a nonempty compact convex subset $X_{0}$ of $X$ and a nonempty compact subset $D$ of $X$ such that for each $x \in X \backslash D$, there exists $y \in X_{0}$ such that $f_{i}\left(y_{i}, x^{i}\right)>t_{i}$ for all $i \in I$.

Then there exists $x \in X$ such that $f_{i}(x)>t_{i}$ for all $i \in I$.

Proof. For each $i \in I$, we define $\psi_{i}: X^{i} \rightarrow 2^{X_{i}}$ by $\psi_{i}\left(x^{i}\right)=\left\{x_{i} \in X_{i}: f_{i}\left(x_{i}, x^{i}\right)>\right.$ $\left.t_{i}\right\}$. Then it is easy to verify that $\left\{\psi_{i}\right\}_{i \in I}$ satisfies all the conditions of Theorem 2.1. The result follows.

The above result generalizes Theorem 3 in [Fan1].

\section{REFERENCES}

[Br] F.E. Browder, The fixed point theory of multi-valued mappings in topological vector spaces, Math.Ann. 177(1968), 283-301. MR 37:4679

[Fan] Ky Fan, Sur un théorème minimax, C.R.Acad.Sci. Paris, 259(1964), 3925-3928. MR 30:5145

[Fan1] Ky Fan, Applications of a theorem concerning sets with convex sections, Math. Ann. 163(1966), 189-203. MR 32:8101

[Fan2] Ky Fan, Some properties of convex sets related to fixed point theorems, Math. Ann. 266(1984), 519-537. MR 85i:47060

[Fan3] Ky Fan, Fixed point and minimax theorems in locally convex linear spaces, Proc. Nat. Acad. Sci. U.S.A. 38(1952), 121-126. MR 13:858d

[Lan] K. Q. Lan, A generalization of $H$-space and some results on multivalued mappings without convexity, J. Math. Anal. Appl. 194 (1995), 511-528. MR 96f:54054

[Ma] T.W. Ma, On sets with convex sections, J. Math. Anal. Appl. 27(1969), 413-416. MR 40:1676

[S.T] M.H. Shih and K.K. Tan, Non-compact sets with convex sections, Pacific J. Math. 119(1985), 473-479. MR 87e:52006

[S.T.K] M.H. Shih and K.K. Tan, Non-compact sets with convex sections, II, J. Math. Anal. Appl. 120(1986), 264-270. MR 87m:52004

[T] E. Tarafdar, On minimax principles and sets with convex sections, Publ. Math. Debrecen 29(1982), 219-226. MR 84a:47074

[T.T] E. Tarafdar and H.B. Thompson, On Ky Fan's minimax principle, J. Aust. Math. Soc. Series A. 26(1978), 220-226. MR 80a:47086

[Ty] A. Tychonoff, Ein Fixpunktsatz, Math Ann. 111 (1935), 767-776.

[Y.P] N.C. Yannelis and N.D. Prabhakar, Existence of maximal elements and equilibria in linear topological spaces, J. Math. Econom. 12(1983), 233-245. MR 87h:90061a

Department of Mathematics, University of Glasgow, Glasgow G12 8QW, United KINGDOM

E-mail address: kl@maths.gla.ac.uk

E-mail address: jrlw@maths.gla.ac.uk 